\title{
VERxVE 144-Week Results: Nevirapine Extended Release (NVP XR) QD Versus NVP Immediate Release (IR) BID with FTC/TDF in Treatment- Na冈ve HIV-1 Patients
}

\section{Brinson $\mathrm{C}^{1 *}$, Bogner $\mathrm{JR}^{2}$, Nelson $\mathrm{M}^{3}$, Podzamczer $\mathrm{D}^{4}$, Quinson $\mathrm{AM}^{5}$, Drulak $\mathbf{M}^{5}$, Andrade-Villanueva $\mathrm{J}^{6}$, Cahn $\mathrm{P}^{7}$, Santiago $\mathrm{S}^{8}$ and Gathe $\mathrm{J}^{9}$} ${ }^{1}$ Central Texas Clinical Research, Austin, TX, USA

${ }^{2}$ University Hospital of Munich, Munich, Germany

${ }^{3}$ St Stephen's AIDS Trust, London, UK

${ }^{4}$ Hospital Universitari de Bellvitge, Barcelona, Spain

${ }^{5}$ Boehringer Ingelheim Pharmaceuticals, Inc, Ridgefield, CT, USA

${ }^{6}$ Universidad de Guadalajara, Guadalajara, Mexico

${ }^{7}$ Fundación Huspéd, Buenos Aires, Argentina

${ }^{8}$ Care Resource, Miami, FL, USA

${ }^{9}$ Therapeutic Concepts, Houston, TX, USA

\begin{abstract}
Background: We report 96- and 144-week follow-up data from VERxVE, demonstrating that nevirapine (NVP) extended release (XR) $400 \mathrm{mg}$ once daily was non-inferior to NVP immediate release (IR) $200 \mathrm{mg}$ twice daily, each administered on a backbone of emtricitabine/tenofovir.

Methods: VERxVE was a double-blind, double-dummy, non-inferiority study in adults with screening viral load (VL) $>1000$ copies $/ \mathrm{mL}$ and CD4+ cell count $<400$ cells $/ \mathrm{mm}^{3}$ (males) or $<250$ cells $/ \mathrm{mm}^{3}$ (females). Randomisation was stratified by baseline $V L$ (copies $/ \mathrm{mL}$ ) $\leq 100,000$ or $>100,000$. The primary endpoint was confirmed virologic response $(<50$ copies $/ \mathrm{mL}$ ) at week 48 . Cochran's statistic incorporating baseline VL strata tested non-inferiority of XR efficacy versus IR. Secondary endpoints included 144-week sustained virologic response and safety.

Results: In all, 1011 patients were randomised and treated with NVP: 736 (XR n=378; IR $n=358$ ) completed 144 weeks. Virologic response was $63.6 \%$ for NVP XR and 58.5\% for NVP IR (adjusted difference of $4.8 \%$ [95\% confidence interval $-1.1 \%$ to $10.8 \%$ ] favouring NVP XR). No significant differences were seen in changes in CD4+ T-cell counts from baseline, virologic failures or total discontinuation rates between treatment arms, regardless of demographic or baseline characteristics.
\end{abstract}

Conclusions: NVP XR demonstrated non-inferior virologic efficacy to NVP IR in treatment-naïve HIV-infected patients and was well tolerated out to week 144, with a safety profile similar to NVP IR.

Keywords: HIV; Nevirapine; Clinical trial; Extended-release formulation; Once-daily dosing

\section{Introduction}

Non-nucleoside reverses transcriptase inhibitor (NNRTI)based antiretroviral (ARV) regimens are important options for the initial treatment of HIV-1 infection. Nevirapine (NVP; Viramune ${ }^{\circledR}$, Boehringer Ingelheim Pharmaceuticals, Ridgefield, CT, USA) was the first NNRTI to be approved by the US Food and Drug Administration in 1996, and NVP-based ARV regimens have been widely used around the world. As a result, the safety and efficacy profiles of NVP are well understood and established in various patient populations.

One of the key benefits of NVP is the lack of untoward effects of this ARV on serum lipid profiles, which have been reported with other anti-HIV drugs. Elevation of serum lipid risk categories can be a significant concern for HIV-infected patients, who tend to have elevated cardiovascular risk profiles as a direct result of viral infection, which can be further aggravated by some ARVs, especially some HIV protease inhibitors.

The extended-release (XR) formulation of NVP $400 \mathrm{mg}$ (NVP $\mathrm{XR}$ ) was developed to allow once-daily (qd) dosing, since this regimen has been shown to increase patient compliance, regimen adherence and therapeutic benefit, especially in homeless or marginally housed populations [1-3].

VERxVE [4] was a double-blind, double-dummy, non-inferiority phase III clinical trial comparing efficacy and safety of the qd formulation of NVP XR $400 \mathrm{mg}$ versus the immediate-release (IR) formulation of NVP (200 mg twice daily [bid]). A total of 1011 patients meeting the screening criteria of viral load (VL) $>1000$ copies $/ \mathrm{mL}$ and CD4+ cell count $<400$ cells $/ \mathrm{mm}^{3}$ (males) or $<250$ cells $/ \mathrm{mm}^{3}$ (females) were randomised. Over 48 weeks (the primary endpoint in this study), the NVP XR formulation demonstrated non-inferior efficacy compared with NVP IR. In addition, both the XR and the IR formulations demonstrated equivalent adverse event (AE) and tolerability profiles at the 48 -week time point.

Herein we report the efficacy and safety results of the 96- and 144week follow-up of NVP XR versus NVP IR antiviral therapy (ART), each administered on a backbone of emtricitabine/tenofovir, in treatment-naïve HIV-1 patients.

*Corresponding author: Cynthia Brinson, Central Texas Clinical Research Austin, TX 78751, Tel: 512-480-9270; E-mail: cbrinson@ctcrcorp.com

Received June 24, 2013; Accepted August 20, 2013; Published August 25, 2013

Citation: Brinson C, Bogner JR, Nelson M, Podzamczer D, Quinson AM, et al. (2013) VERxVE 144-Week Results: Nevirapine Extended Release (NVP XR) QD Versus NVP Immediate Release (IR) BID with FTC/TDF in Treatment-Naïve HIV-1 Patients. J AIDS Clin Res 4: 233. doi:10.4172/2155-6113.1000233

Copyright: $(2013$ Brinson C, et al. This is an open-access article distributed under the terms of the Creative Commons Attribution License, which permits unrestricted use, distribution, and reproduction in any medium, provided the original author and source are credited. 
Citation: Brinson C, Bogner JR, Nelson M, Podzamczer D, Quinson AM, et al. (2013) VERxVE 144-Week Results: Nevirapine Extended Release (NVP XR) QD Versus NVP Immediate Release (IR) BID with FTC/TDF in Treatment-Naïve HIV-1 Patients. J AIDS Clin Res 4: 233. doi:10.4172/21556113.1000233

Page 2 of 4

\section{Methods}

The details of the methods used in the VERxVE study have been described in detail in the 48-week report [4], and are herein described briefly. VERxVE, a randomised, double-blind, double-dummy non-inferiority ( $-10 \%$ margin), parallel-group, active-controlled trial in adult, ART-naïve, HIV-1 infected patients, compared the efficacy and safety of NVP XR (400 mg qd) with NVP IR (200 mg bid), both administered in combination with emtricitabine/tenofovir once-daily. Eligible patients were stratified by baseline HIV-1 viral load into two groups 1) low $\leq 100,000$ copies $/ \mathrm{mL}$, and 2) high $>100,000$ copies $/ \mathrm{mL}$. Patients who successfully completed the 14-day lead-in period during which they received NVP IR (200 $\mathrm{mg}$ qd) were randomised to either NVP XR 400 mg qd or NVP IR 200 mg bid (Figure 1).

The treatment period was 48 weeks (primary endpoint), with a blinded extension to 144 weeks, which continued after each patient had completed the week 48 visit.

At the conclusion of the 144-week blinded treatment period, patients had the option to enter the open-label extension phase, where they received NVP XR and continued emtricitabine/tenofovir qd treatment (Figure 1). Open-label extension participants visited the clinic every 12 weeks until the last patient enrolled in the trial completed the 144-week blinded treatment phase. At that point, each patient was scheduled for an end-of-trial visit (within 30 days) to gather final long-term safety and efficacy data.

The primary endpoint for VERxVE was confirmed virologic response at week 48. The secondary endpoints included sustained virologic response (SVR), using lower limit of quantification [LLOQ] of 50 copies $/ \mathrm{mL}$, and safety outcomes at weeks 96 and 144 . Virologic response was defined as two consecutive VL measurements $<50$ copies $/ \mathrm{mL}$ (at least 2 weeks apart). In order to be considered an SVR, there could be no viral rebound or change in ART through week 144.

The major criteria for patient inclusion were: (i) adult ( $\geq 18$ years of age) HIV-1-infected patients with no prior ART; (ii) CD4+ counts $>50$ to $<400$ copies $/ \mathrm{mL}$ in males or $>50$ to $<250$ copies/mL in females; (iii) baseline HIV-1 VL $\geq 1000$ copies/mL; and (iv) Karnofsky score $>70$.

The Cochran's statistic with variance correction was used to calculate the difference and $95 \%$ confidence interval (CI) between the two NVP formulations (XR vs. IR). The non-inferior efficacy of NVP XR versus NVP IR was determined by a lower limit of the CI greater than $-10 \%$. All of the safety parameters reported here are presented descriptively.

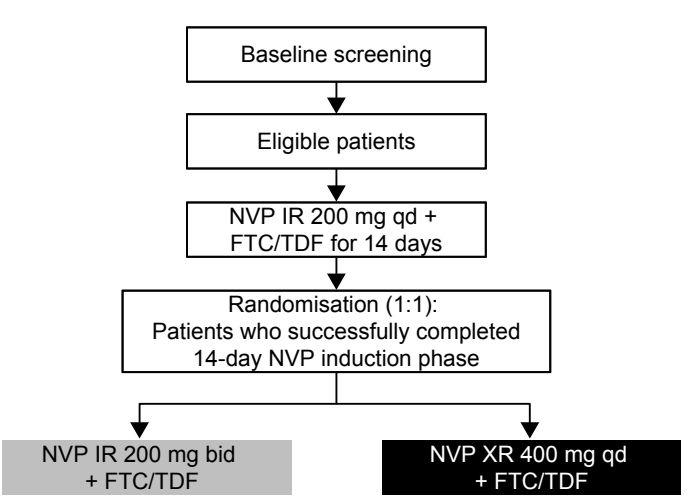

Figure 1: Study design of the VERxVE trial. bid, twice daily; FTC/TDF, emtricitabine/tenofovir; NVP, nevirapine; qd, once daily.

\begin{tabular}{|c|c|c|}
\hline & NVP IR & NVP XR \\
\hline Baseline HIV $\left(\log _{10}\right.$ copies $\left./ \mathrm{mL}\right), n$ & $\boldsymbol{n}=\mathbf{5 0 8}$ & $\boldsymbol{n}=\mathbf{5 0 5}$ \\
\hline Mean & & \\
\hline SD & 4.7 & 4.7 \\
\hline Median & 0.6 & 0.7 \\
\hline Baseline HIV RNA (copies/mL), $n(\%)$ & 4.7 & 4.7 \\
\hline$<1,000$ & & $3(0.6)$ \\
\hline 1,000 to 10,000 & $2(0.4)$ & $77(15.2)$ \\
\hline$>10,000$ to 100,000 & $250(49.2)$ & $260(51.5)$ \\
\hline$>100,000$ to $1,000,000$ & $167(32.9)$ & $155(30.7)$ \\
\hline$>1,000,000$ & $7(1.4)$ & $10(2.0)$ \\
\hline Baseline CD4+ T-cell count, cells $/ \mathrm{mm}^{2}$ & & \\
\hline$n$ & 507 & 503 \\
\hline Mean & 227.7 & 229.6 \\
\hline SD & 85.9 & 81.4 \\
\hline Median & 224.5 & 227.5 \\
\hline
\end{tabular}

NVP IR, nevirapine immediate release; NVP XR, nevirapine extended release; SD standard deviation

Table 1: Patient demographics and baseline characteristics.

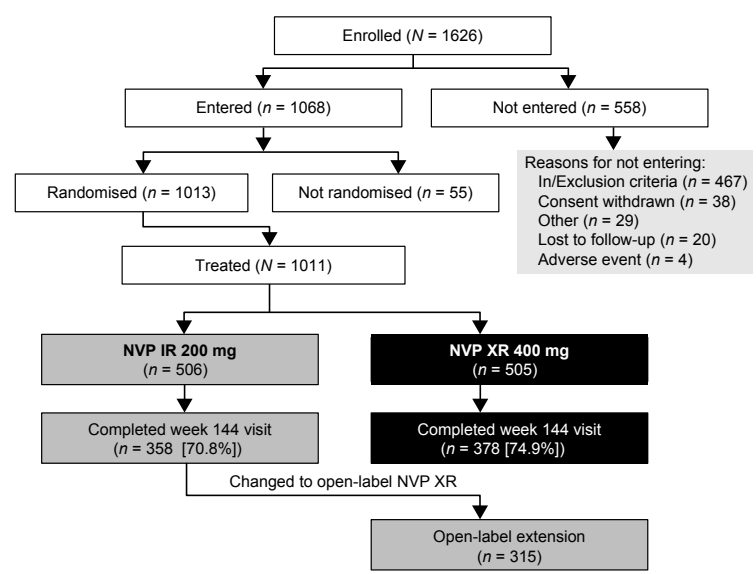

Figure 2: Patient disposition through week 144 and beyond in the openlabel extension. NVP IR, nevirapine immediate release; NVP XR, nevirapine extended release; $X R$, extended release.

\section{Results}

\section{Patients}

Baseline characteristics, including mean HIV VL, proportion of patients in each VL category and mean CD4+ T-cell count, were similar between the two treatment arms (Table 1) [4]. VERxVE study results at 48 weeks have been previously reported [4].

\section{Patient disposition at 144 weeks}

At 144 weeks, $70.8 \%(n=358 / 506)$ of patients originally on the IR formulation were still receiving NVP IR and $74.9 \%(n=378 / 505)$ who were originally receiving the XR formulation remained on the XR formulation. Medication discontinuation was similar in both groups, wherein $29.2 \%(n=148 / 506)$ of patients discontinued NVP IR and $25.1 \%(n=127 / 505)$ of patients discontinued NVP XR. Patients in the IR arm who completed the 144 weeks of treatment were given the option of switching to the extended-release formulation (NVP XR) and continuing in the open-label extension of the study. In all, 315 patients in the IR arm exercised this option (Figure 2). 
Citation: Brinson C, Bogner JR, Nelson M, Podzamczer D, Quinson AM, et al. (2013) VERxVE 144-Week Results: Nevirapine Extended Release (NVP XR) QD Versus NVP Immediate Release (IR) BID with FTC/TDF in Treatment-Naïve HIV-1 Patients. J AIDS Clin Res 4: 233. doi:10.4172/21556113.1000233

Page 3 of 4

\section{Efficacy}

The SVR, based on an LLOQ of 50 copies/mL by time to loss of virologic response (TLOVR; full analysis set), was similar between the two arms at each time point of the study; however, both groups demonstrated declining SVR rates over the long-term follow-up, from week 48 to week 144 (Figure 3). At week 144, SVR was achieved in 63.56\% ( $\mathrm{n}=321 / 505)$ of patients receiving NVP XR qd formulation, compared with $58.5 \%$ ( $n=296 / 506)$ of patients receiving the NVP IR bid formulation.

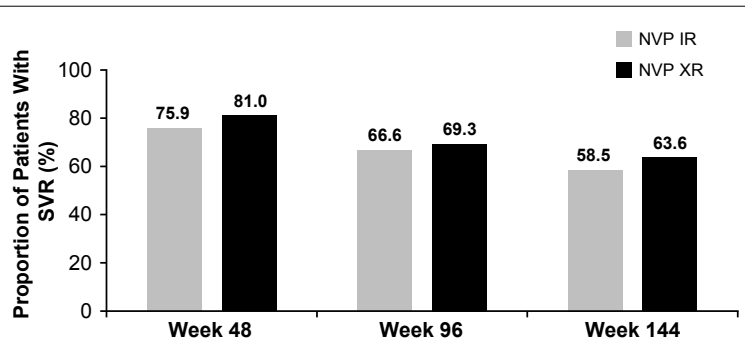

Figure 3: Proportion of patients with sustained virologic response at weeks 48, 96 and 144, by TLOVR. NVP IR, nevirapine immediate release; NVP XR, nevirapine extended release; SVR, sustained virologic response; TLOVR, time to loss of virologic response.

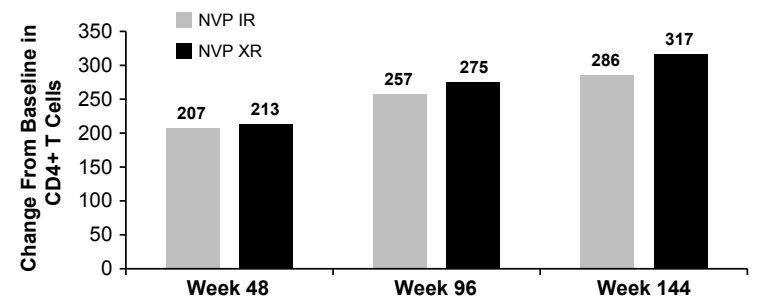

Figure 4: Changes in CD4+ T-cell counts from baseline at weeks 48, 96 and 144.
At week 144, the adjusted difference in the proportion of SVR for the XR and IR treatment groups was $4.84 \%$ (95\% CI $-1.11 \%$ to $10.79 \%$ ) favouring NVP XR. The lower bound of CI excluded the $-10 \%$ non-inferiority margin $(\mathrm{P}<0.0001$ for the non-inferiority test). By SNAPSHOT analysis, the adjusted difference in SVR proportions between the XR and IR formulations was $4.08 \%$ (95\% CI $-1.75 \%$ to 9.91\%), favouring NVP XR.

At week 144, no associations between patient demographics (age group, gender, race, ethnicity or region) or patient baseline characteristics (CD4+ count, HIV subtype and CDC class) were associated with SVR in either treatment group. Patients with lower VL $(<100,000)$ at baseline generally achieved a higher rate of SVR versus patients with higher VL $(>100,000)$ at baseline, for both NVP formulations.

By TLOVR analysis, no clinically important differences were noted in the change in VL from week 48 to week 144 results between the IR and XR NVP formulations, i.e. results achieved by week 48 were generally maintained to week 144 .

Patients in both treatment arms showed improvement in their CD4+ T-cell counts over the course of the trial, and this positive trend continued through 144 weeks. As seen in Figure 4, progressive increases from baseline in CD4+ T-cell counts with IR versus XR treatment, respectively, were seen at weeks 48 (207 vs. 213), 96 (257 vs. 275) and 144 (286 vs. 317 ).

\section{Safety and tolerability out to week 144}

The overall safety and tolerability results in patients receiving both the XR and the IR formulations of NVP at week 144 were similar to results reported at weeks 48 and 96 . It is important to note that the rate of AEs, drug-related AEs (investigator-defined), serious AEs and Division of Acquired Immunodeficiency Syndrome (DAIDS) grade 3 and grade 4 AEs were similar between the two treatment groups out to week 96 and week 144 (Table 2).

\begin{tabular}{|c|c|c|c|c|c|}
\hline & \multicolumn{2}{|c|}{$\begin{array}{l}\text { Up to week } 96 \\
\text { FAS }\end{array}$} & \multicolumn{2}{|c|}{$\begin{array}{l}\text { Up to week } 144 \\
\text { FAS }\end{array}$} & \multirow{2}{*}{$\begin{array}{l}\text { IR patients in open-label extension } \\
\text { (post- week 144) } \\
\text { NVP XR } \\
400 \text { mg qd } \\
\mathrm{N}=315 \\
n(\%)\end{array}$} \\
\hline & $\begin{array}{c}\text { NVP } \\
\text { IR } 200 \text { mg bid } \\
N=506 \\
n(\%)\end{array}$ & $\begin{array}{c}\text { NVP XR } \\
400 \mathrm{mg} \text { qd } \\
\mathrm{N}=505 \\
n(\%)\end{array}$ & $\begin{array}{c}\text { NVP } \\
\text { IR } 200 \mathrm{mg} \text { bid } \\
\mathrm{N}=\mathbf{5 0 6} \\
n(\%)\end{array}$ & $\begin{array}{c}\text { NVP XR } \\
400 \mathrm{mg} \text { qd } \\
\mathrm{N}=505 \\
n(\%)\end{array}$ & \\
\hline Any AE & $470(92.9)$ & $459(90.9)$ & $472(93.3)$ & $471(93.3)$ & $141(44.8)$ \\
\hline Investigator-defined drug-related $\mathrm{AE}$ & $134(26.5)$ & $109(21.6)$ & $135(26.7)$ & $118(23.4)$ & $5(1.6)$ \\
\hline AEs leading to discontinuation of trial drug & $52(10.3)$ & $44(8.7)$ & $54(10.7)$ & $43(8.5)$ & $0(0.0)$ \\
\hline Serious AEs* & $76(15.0)$ & $78(15.4)$ & $83(16.4)$ & $93(18.4)$ & $9(2.9)$ \\
\hline Fatal & $5(1.0)$ & $3(0.6)$ & $7(1.4)$ & $3(0.6)$ & $0(0.0)$ \\
\hline Immediately life-threatening & $3(0.6)$ & $3(0.6)$ & $5(1.0)$ & $5(1.0)$ & $0(0.0)$ \\
\hline Disability/incapacity & $0(0.0)$ & $0(0.0)$ & $1(0.2)$ & $1(0.2)$ & $1(0.3)$ \\
\hline Required hospitalisation & $65(12.8)$ & $68(13.5)$ & $71(14.0)$ & $81(16.0)$ & $8(2.5)$ \\
\hline Prolonged hospitalisation & $4(0.8)$ & $3(0.6)$ & $4(0.8)$ & $4(0.8)$ & $0(0.0)$ \\
\hline Congenital anomaly & $0(0.0)$ & $0(0.0)$ & $0(0.0)$ & $0(0.0)$ & $0(0.0)$ \\
\hline Other & $9(1.8)$ & $10(2.0)$ & $11(2.2)$ & $12(2.4)$ & $1(0.3)$ \\
\hline DAIDS grade 3 or 4 AEs & $112(22.1)$ & $94(8.6)$ & $124(24.5)$ & $111(22.0)$ & $8(2.5)$ \\
\hline DAIDS grade 4 AEs & $26(5.1)$ & $21(4.2)$ & $28(5.5)$ & $25(5.0)$ & $3(1.0)$ \\
\hline Study drug-related DAIDS grade 3 or 4 AEs & $38(7.5)$ & $28(5.5)$ & $39(7.7)$ & $29(5.7)$ & $0(0.0)$ \\
\hline Study drug-related DAIDS grade 4 AEs & $11(2.2)$ & $7(1.4)$ & $11(2.2)$ & $7(1.4)$ & $0(0.0)$ \\
\hline
\end{tabular}

*A patient may be counted in more than one seriousness criterion.

Percentages are calculated using total number of patients per treatment as the denominator.

AE, adverse event; bid, twice daily; DAIDS, Division of Acquired Immunodeficiency Syndrome; FAS, full analysis set; NVP IR, nevirapine immediate release; NVP XR, nevirapine extended release; qd, once daily

Table 2: Summary of overall adverse events: up to week 96 and 144 FAS (randomized phase), and open-label extension (beyond week 144). 
With regard to severity of AEs, the majority of patients in both treatment groups reported either mild or moderate events. The frequency of patients reporting liver abnormalities or rashes did not change in any clinically meaningful way at the week 48, 96 and 144 follow-up visits.

Up to week 96 there were a total of $4(0.4 \%, \mathrm{~N}=1,011)$ of patients with Stevens-Johnson syndrome (investigator defined as drug related), all of which were in the nevirapine IR group. This represents an increase of one patient since the Week 48 assessment, where three cases were noted. No new cases of Stevens Johnson syndrome were reported after week 96

There were a total of 16 deaths in the VERxVE trial; none were attributed to study medication. Ten deaths were reported in the first 48 weeks, four deaths were reported between 48 and 96 weeks and two additional deaths reported after 96 weeks.

It is important to note that no novel or additional safety findings were reported after the 48-week time point and up to weeks 96 and 144 .

\section{Safety and tolerability beyond week 144: the open-label extension}

In the open-label extension phase of the trial, when patients who had been on the IR formulation of NVP for 144 weeks had the option of switching to the XR qd formulation, the rate of all reported AEs, including drug-related AEs (investigator defined), serious AEs and DAIDS grade 3 and grade 4 , were all lower in patients in the open-label extension.

\section{Discussion}

A particular strength of this trial was the double-blind, doubledummy design, which ensured complete blinding of patients to the investigators and sponsor staff prior to week 48. Patients and investigators continued to be blinded to treatment group assignments after week 48 .

In all, 1011 patients in VERxVE successfully completed NVP lead-in dosing, and began the randomised treatment period. The demographic data and baseline characteristics were also well balanced between patients randomised into the treatment groups.

The non-inferior efficacy of the XR formulation of NVP for the treatment of HIV-1 infection in patients who began ART for the first time was established at the week 48 analysis of virologic response [4]. The results at this time point also indicated similar safety and tolerability between the two treatment arms, with similar rates of all reported AEs, including drug-related AEs (investigator-defined), serious AEs and DAIDS grade 3 and grade 4 AEs, between the two treatment arms [4].

The present report describes long-term follow-up data from these patients after 96 and 144 weeks of treatment. These data demonstrate that the XR formulation of NVP ( $400 \mathrm{mg}$ qd) continued to demonstrate virologic non-inferiority compared with the IR formulation $(200 \mathrm{mg}$ bid) as measured by SVR (VL $<50$ copies $/ \mathrm{mL}$ ). The efficacy results presented here continue to demonstrate that NVP XR is non-inferior to NVP IR with regard to HIV-1 VL suppression at up to and beyond week 144 in ART-naïve HIV-1-positive patients.

In addition, at week 144, the XR qd formulation continued to be well tolerated, with a safety profile equivalent to that seen with the IR bid formulation over the same follow-up time course. No significant or meaningful differences were observed between the two treatment arms with regard to TLOVR, change from baseline in HIV-1 VL at week
144, change in CD4+ cell count from baseline at week 144 or time to emergence of new AIDS-defining events, AIDS-related progression of events or death.

With regard to the long-term safety, i.e. efficacy and tolerability of NVP-based regimens, patients in the open-label extension phase of the trial (who had already completed 144 weeks of NVP IR treatment and had the option of switching to the XR qd formulation), demonstrated that the rate of reported AEs, including drug-related AEs (investigator defined), serious AEs and DAIDS grade 3 and grade 4 AEs, were all lower in patients in the open-label extension.

Once-daily formulations of antiretroviral medications can be especially advantageous for the management of HIV-infected patients from disadvantaged populations, including homeless or marginally housed patients [5]. In these populations, the convenience of oncedaily administration significantly enhances patient adherence to ARV regimens, which has been shown to reduce the incidence of virologic breakthrough, and the emergence of virologic resistance [5].

The 96 - 144 week data presented in this paper strongly suggest the long-term durability of NVP XR-based regimens for treatment-naïve HIV-1 patients.

\section{Acknowledgments}

This work was supported by Boehringer Ingelheim Pharmaceuticals, Inc (BIPI). Writing, editorial support and formatting assistance was provided by José L. Walewski, PhD, of Envision Scientific Solutions, which was contracted and compensated by BIPI for these services.

\section{Disclosures}

The authors meet criteria for authorship as recommended by the International Committee of Medical Journal Editors (ICMJE) and were fully responsible for all content and editorial decisions, and were involved at all stages of manuscript development. The authors received no compensation related to the development of the manuscript, but are consultants for Boehringer Ingelheim Pharmaceuticals Inc (BIPI) and received research grant support from BIPI for this study.

\section{Dr Quinson and Dr Drulak are employees of BIPI.}

Dr Brinson is a consultant with Gilead Sciences, Inc.

Dr Gathe is a consultant with Gilead Sciences Inc, GlaxoSmithKline Inc., Pfizer Inc, Bristol-Myers Squibb Company, Abbott Laboratories, Janssen Pharmaceuticals Inc., Roche, Merck and Co., Tibotec Therapeutics and ViiV Healthcare.

Dr Bogner discloses his role as a speaker for most all manufactures of anti-HIV compounds, and has participated in AD-Boards with Abbott Laboratories, Janssen Pharmaceuticals Inc. and BIPI.

Dr Andrade-Villanueva, Dr Cahn, Santiago, Dr Podzamczer and Dr Nelson do not have any relationships to disclose.

\section{References}

1. Claxton AJ, Cramer J, Pierce C (2001) A systematic review of the associations between dose regimens and medication compliance. Clin Ther 23: 1296-1310.

2. Osterberg L, Blaschke T (2005) Adherence to medication. N Engl J Med 353 487-497.

3. Parienti JJ, Bangsberg DR, Verdon R, Gardner EM (2009) Better adherence with once-daily antiretroviral regimens: a meta-analysis. Clin Infect Dis 48: 484488.

4. Gathe J, Andrade-Villanueva J, Santiago S, Horban A, Nelson M et al (2011) Efficacy and safety of nevirapine extended-release once daily versus nevirapine immediate-release twice-daily in treatment-naive HIV-1-infected patients. Antivir Ther 16: 759-769.

5. Bangsberg DR, Ragland K, Monk A, Deeks SG (2010) A single tablet regimen is associated with higher adherence and viral suppression than multiple tablet regimens in HIV+ homeless and marginally housed people. AIDS 24: 2835 2840 . 